\title{
A Comparative Study on the Model of PM2.5 Direct or Indirect Interaction with Bronchial Epithelial Cells.
}

\section{Yan Wang}

Capital Medical University

\section{Xin Zuo}

Chaoyang District Center for Disease Control and Prevention

\section{Fuyang Jiang}

Capital Medical University

\section{Lin Hou}

Capital Medical University

Qiyue Jiang

Capital Medical University

Zhonghui Zhu

Capital Medical University

LIN TIAN ( $\square$ tian_lin@163.com )

Capital Medical University https://orcid.org/0000-0001-6488-7999

\section{Research Article}

Keywords: PM2.5, epithelia, co-culture, inflammation

Posted Date: May 24th, 2021

DOI: https://doi.org/10.21203/rs.3.rs-482547/v1

License: (c) (1) This work is licensed under a Creative Commons Attribution 4.0 International License. Read Full License

Version of Record: A version of this preprint was published at Environmental Science and Pollution Research on January 30th, 2022. See the published version at https://doi.org/10.1007/s11356-02118324-2. 


\section{Abstract}

The impact of PM2.5 on epithelial cells is a pivotal process leading to many lung pathological changes and pulmonary diseases. In addition to PM2.5 direct interaction with epithelia, macrophages that engulf PM2.5 may also influence the function of epithelial cells. However, among the toxic researches of PM2.5, there is a lack of evaluation of direct or indirect exposure model on human bronchial epithelial cell against PM2.5. In this present research, PM2.5-exposed human bronchial epithelial cell line (BEAS-2B) serves as the direct interaction model, while the contrast is to indirect stimulation model, which takes advantage of transwell co-culture system to carry out that PM2.5 is promptly contacted with macrophages rather than BEAS-2B. By comparing these two modes of interaction, we determined the viability of BEAS-2B and mRNA and/or protein expression profile of transcription factors Nrf2,NF-kB and according inflammatory indicators, with a view to evaluating the effects of different interaction modes of PM2.5 on epithelial cell damage in vitro. We have found that macrophage involvement may protect epithelia from PM2.5 cytotoxic effect, while strengthen the inflammation response.

\section{Introduction}

PM2.5 is a sort of airborne particles and droplets with small particle size, long sustained in many developing countries. It easily absorbs hazardous substances in the environment, entering into the respiratory tract and even across blood-gas barrier, prompting complex and harmful biological effects. Lung tissue is the initial site of PM2.5 deposition and one of its toxicity targets. In many previous in vitro studies, researchers prefer to study the effect of PM2.5 on one or more certain lung cells (Kumar et al. 2021). Yet, the entrance of PM2.5 into the lung and functioning has been proven to be an intricate biological process. When PM2.5 is inhaled into the lung tissue through the respiratory tract and reaches the end of the bronchi and alveoli, it can directly act on the surface of the tissue cells to undermine physical functions. At the same time, some of PM2.5 particles can also be engulfed by macrophages to produce cytokines, thereby affecting surrounding bronchial epithelia cells. A recent study has found that quantification of leukocyte population in mice lung $24 \mathrm{~h}$ post-PM2.5 treatment showed significant increase of macrophages (Jeong et al. 2019). Therefore, PM2.5 may have an alternative mode of interaction with bronchial epithelia cells after entering the lung tissue comparing with the directly touch. To date, the difference between the direct and indirect effects of PM2.5 on human bronchial epithelia cells in vitro is not well known.

The mounting evidences have proved that the adverse effects caused by PM2.5 to lung tissue involve intracellular oxidative stress and inflammatory responses (Feng et al. 2016). PM2.5-induced reactive oxygen species (ROS) is considered to be an essential mediator of PM2.5 toxicity (Cho et al. 2018). In the previous research, we have also confirmed that the ROS content was gradually increased with the stimulation of PM2.5 in bronchial epithelia cells (Zhu et al. 2019). The increasing ROS can impair the antioxidant system, eventually giving rise to cellular oxidative stress. Erythiord-2-related factor 2 (Nrf2), which often serves as a transcription factor, regulates the transcription of the enzymes involved in phase I and phase II detoxification of exogenous and endogenous products (Tonelli et al. 2018). Additional, it is 
also acknowledged that oxidative stress stemming from PM2.5 can facilitate NF-kB transcription, stimulating inflammatory cytokine synthesis (Falcon-Rodriguez et al. 2016). Using Nrf2 and NF-KB as a sensor marker to study the effects of poisons on oxidative stress and inflammation in vitro has great implications.

In order to better simulate the impairment of PM2.5 to lung tissue, we leverage direct stimulation cell model and indirect stimulation cell model respectively. At present, most of the in vitro studies adopted PM2.5 to stimulate the test cells directly. In this present research, we compared direct model with indirect stimulation model, which took advantage of transwell co-culture system with free exchange of cytokines across the transwell membrane, while restricting direct PM2.5-epithelia cell contact. By comparing these two modes of action, we determined mRNA and/or Protein expression profile of transcription factors Nrf2, $\mathrm{NF}-\mathrm{kB}$ and related inflammatory indicators in stimulated bronchial epithelial cells, with a view to evaluating the effects of different interaction modes of PM2.5 on cell damage in vitro.

\section{Materials And Methods}

\section{1 $\mathrm{PM}_{2.5}$ collection and preparation.}

$\mathrm{PM}_{2.5}$ collection and preparation have been performed as description before (Li et al. 2020). Briefly, largevolume air particles sampler was used to collect PM samples at the location of Capital Medical University with the height of 15 meters from October 2017 to March 2018. The specific parameters of sampler were set to a flow rate of $1.05 \mathrm{~m}^{3} / \mathrm{min}$, the sampling temperature around -10 to $10^{\circ} \mathrm{C}$, and with $25-45 \%$ relative humidity.

The filter was collected every 24 -hour and stored at the temperature of $-80^{\circ} \mathrm{C}$. During elution, the collected PM2.5 filter was cut into a size of $1 \mathrm{~cm}^{2}$, and ultrasonically oscillated in appropriate amount of sterilized ultrapure water for $15 \mathrm{~min} 3$ times. The eluate was collected, filtered through 6-8 layers of sterile gauze, and the filtrate was frozen at $-80^{\circ} \mathrm{C}$ overnight and lyophilized under vacuum to dry the powder. The dried PM2.5 was collected in a closed glass container and stored in a dark environment at $-80^{\circ} \mathrm{C}$.

\subsection{Assessment of PM chemical composition}

The PAHs in PM2.5 were analyzed by gas chromatography-mass spectrometry (7000C, Agilent Technologies, Santa Clara, USA). The heavy metals in PM2.5 were analyzed by inductively coupled plasma-mass spectrometry (8800, Agilent, USA). As a control, blank glass fiber filters were vacuum-freeze dried and weighed in parallel with the PM2.5 samples. The concentrations of PAHs and metals in the blank glass fiber filter were determined and deducted from the value of PAHs and metals in the PM2.5 glass fiber filter as background.

\subsection{Cell treatment}

Human bronchial epithelial cells (BEAS-2B), human mononuclear cells (THP-1) were purchased from Peking Union Medical College, Cell Bank (Beijing, China). BEAS-2B cells were cultured in DMEM medium 
supplemented with 10\% fetal bovine serum (HyClone) and 1\% penicillin and streptomycin (Thermo Fisher Scientific). THP-1 cells were cultured in RPMI1640 medium (HyClone) with 10\% fetal bovine serum and $1 \%$ penicillin and streptomycin. The cells were routinely cultured in a humidified atmosphere of $5 \% \mathrm{CO}_{2}$ at $37^{\circ} \mathrm{C}$.

In the model of direct interaction, BEAS-2B cells were cultured for three generations and planted into 6well plate. Cells with $70-80 \%$ confluency were treated with different concentrations of PM2.5 ranging from 0 to $200 \mu \mathrm{g} / \mathrm{mL}$. In tems of indirect interaction, to obtain differentiated macrophages from THP-1 cells, THP-1 monocytes were firstly incubated with PMA $(50 \mathrm{ng} / \mathrm{mL})$ for $48 \mathrm{~h}$ and then planted into the upper chamber of co-culture transwell. Subsequently, THP-1 cells were stimulated by the corresponding concentrations of PM2.5 and incubated with BEAS-2B cells which were planted into the lower chamber. BEAS-2B cells were harvested in both models 24-hour incubation afterward.

\subsection{Identification of THP-1 cells differentiation by flow cytometry}

The identification and characterization of THP-1 cells differentiation were performed using flow cytometry. THP-1 cells were harvested after PMA stimulation and incubated with PE-conjugated mouse anti-human CD14, FITC-conjugated mouse anti-human CD68, or a mouse IgM/lgG2a isotype control for 60 min. After washing the cells with PBS, the expression of the cell surface markers CD14 and CD68 was analyzed using flow cytometry on a FACSCalibur ${ }^{\text {TM }}$ (BD Biosciences).

\subsection{MTT assay.}

Cell viability was evaluated by MTT assay. BEAS-2B cells were plated into 96-well microtiter plates at a density of 5000 cells/well. After $24 \mathrm{~h}$, the cells were treated with PM2.5 or supernatant from PM2.5treated THP-1 cells. MTT solution ( $5 \mathrm{mg} / \mathrm{mL}$ in phosphate buffered saline; PBS) was added to each well after $24 \mathrm{~h}$ incubation and then the cells were incubated for $2 \mathrm{~h}$. The medium was removed and DMSO was added to dissolve the MTT formazan. The absorbance was measured at $570 \mathrm{~nm}$ while using a microplate reader

\subsection{Quantitative polymerase chain reaction (qPCR) assay.}

For qRT-PCR, BEAS-2B cells in both models were lysed using TRIzol (Invitrogen). RNA was extracted and reverse transcribed into cDNA using SuperScript III (Invitrogen). qRT-PCR was performed using Thermo Hybaid. The primers used for qRT-PCR analysis of human RNA include: Nrf2: 5'-

AACCACCCTGAAAGCACAGC-3' and 5'-TGAAATGCCGGAGTCAGAATC-3'; NF-KB: 5'AACAGCAGATGGCCCATACCT-3' and 5'-ACGCTGAGGTCCATCTCCTTG-3'; TNF-a: 5'-

TCTTCAGCTCTGGGGAAAATGC-3' and 5'-TTTTGCTTGCCTCCCCAGG-3'; IL-1: 5'-

GGATATGGAGCAACAAGTGG-3' and 5'-ATGTACCAGTTGGGGAACTG-3'; IL-6: 5'-

TAGCCGCCCCACACAGACAG-3' and 5'-GGCTGGCATTTGTGGTTGGG-3'; IL-8: 5'-

GAGGGTTGTGGAGAAGTTTTTG-3' and 5'-CTGGCATCTTCACTGATTCTTG-3'; GAPDH: 5'-

TCAACGACCACTTTGTCAAGCT-3' and 5'-CCATGAGGTCCACCACCCT-3'. 


\subsection{Western blotting}

BEAS-2B cells lysates in both models were prepared in radioimmunoprecipitation assay buffer supplemented with protease and phosphatase inhibitors (NanJing KeyGen Biotech Co.,Ltd.). Protein concentrations were determined using a bicinchoninic acid (BCA) protein assay kit (Thermo Fisher Scientific, Inc.). The protein samples $(25 \mu \mathrm{g})$ were separated by $10 \%$ SDS-PAGE and were transferred onto polyvinylidene difluoride membranes (Millipore Sigma). The membranes were blocked with $5 \%$ non-fat milk in Tris-buffered saline at room temperature for $1 \mathrm{~h}$. Subsequently, the membranes were incubated with the following antibodies (1:1,000 dilutions) overnight at $4^{\circ} \mathrm{C}$ : Nrf2(ab31163, Abcam), NF-kB p65 88242, CST $₫$, and GAPDH $\otimes 2118, C S T \rrbracket$. Horseradish peroxidase-conjugated anti-rabbit $(7074$, CST) were used as secondary antibodies (1:5,000 dilutions), and blots were incubated with them at room temperature for $2 \mathrm{~h}$. ImageJ software was used to semi-quantify the relative expression levels of the target proteins, which were normalized to the respective internal controls.

\subsection{Statistical analysis}

All experiments were performed in triplicate. Derived values are presented as means \pm SD. Comparison of means among multiple groups was accomplished by one-way analysis of variance (ANOVA). A multiplerange least significant difference (LSD) was used for inter-group comparisons. $P$ values $<0.05$ were considered statistically significant. All statistical analyses were performed with SPSS 17.0.

\section{Results}

\subsection{Chemical composition analysis of PM2.5}

We mainly examined the aromatic compounds and inorganic elements in collecting PM2.5 particles (Fig. 1). Analysis of aromatic compounds in PM2.5 illustrated that the highest detection concentration was seen within benzo[b] fluoranthene $\left(204.5 \pm 5.02 \mathrm{ng} / \mathrm{m}^{3}\right)$ followed by benzo[k] fluoranthene (176.9 \pm $\left.8.11 \mathrm{ng} / \mathrm{m}^{3}\right)$ and chrysene $\left(148.3 \pm 16.62 \mathrm{ng} / \mathrm{m}^{3}\right)$. Analysis of inorganic elements in PM2.5 revealed the predominance of $\mathrm{Zn}\left(911.5 \pm 26.10 \mathrm{ng} / \mathrm{m}^{3}\right), \mathrm{Pb}\left(361.1 \pm 2.02 \mathrm{ng} / \mathrm{m}^{3}\right), \mathrm{Mn}\left(308.5 \pm 6.23 \mathrm{ng} / \mathrm{m}^{3}\right)$, and $\mathrm{Cu}$ $\left(292.6 \pm 10.57 \mathrm{ng} / \mathrm{m}^{3}\right)$.

\subsection{THP-1-M $\varphi$ cell differentiation.}

Before implantation in the Transwell upper chamber, THP-1 cells were induced by phorbol-12-myristate13-acetate (PMA) to differentiate into adherent macrophages, as outlined in the methods section. THP-1 cells were single suspension cultured cells with complete morphology, uniform size and good refractive index (Fig. 2A). After 48 hours of stimulation with PMA (50ng/mL), THP-1 cells were induced to differentiate into macrophages (THP-1-M $\varphi$ ) instead of suspended growth. Most of the differentiated cells were still round or oval, the phenomenon of cell aggregation was more obvious (Fig. 2B). The cell surface proteins of monocytic cell such as CD14 and CD68 were detected by flow cytometry. The results showed that human monocytes THP-1 cells expressed high levels of CD14, while THP-1-derived macrophages 
expressed low CD14. Additionally, CD68 is a very classic marker and presenting a high expression both in THP-1 and THP-1-M $\varphi$ cells (Fig. 2C). Combining with the morphological changes and adherent growth, it showed that monocytes had successfully differentiated into macrophages.

\subsection{Cell viability in both models.}

The effect of PM2.5 on cell viability was evaluated by MTT assay and the result displaying in Fig. 3B illustrated that with the increasing concentration of PM2.5, the cell viability decreased accordingly. It is noted that cell proliferation showed more significant inhibition with the direct exposure model compared with the indirect model.

\subsection{Expressions of Nrf2 and NF-KB in both models.}

Beas-2B cells were harvested from direct or indirect model individually, which schematic diagram is shown in Fig. 3A. Using different concentrations of PM2.5 to directly or indirectly stimulate BEAS-2B cells for 24 hours, we observed mRNA and protein expression levels of the transcription factor Nrf2 and NF-kB. With the increasing concentration of PM2.5, mRNA and protein expression of Nrf2 were increased and the highest expression levels appeared in the $25 \mu \mathrm{g} / \mathrm{mL}$ group, which was significantly different from the control group in both model. Direct contact with PM2.5 lead to Nrf2 mRNA level increase dramatically, while there was no significant different tendency among direct and indirect model at the protein level. In terms of transcription factor NF-kB, direct stimulation of PM2.5 could upregulate the expression of NF-kB in a dose-dependent manner with expression peak of $50 \mu \mathrm{g} / \mathrm{mL}$ (Fig. 4). Despite a similar dose-dependent expression of NF-kB in the indirect stimulation model whether in gene or protein profile, we found that the peak expression of mRNA and protein of NF-kB shift to higher concentration group of PM2.5 (100 $\mu \mathrm{g} / \mathrm{mL})$.

\subsection{Differential expressions of inflammatory factors in both models.}

Next, we had examined the mRNA expressions of inflammatory factors, which are downstreams of NFKB. Compared to the control groups, PM2.5-exposure displayed an obvious increase inflammatory factors such as IL-1, IL-6, and IL-8 in either direct or indirect stimulation model (Fig. 5). Specifically, in direct model IL-1 mRNA expression reached its peak in $50 \mu \mathrm{g} / \mathrm{mL}$ PM2.5-exposure group. Interestingly, we found that in the indirect exposure model, IL-1, IL-6, and IL-8 mRNA expressions were strengthen in the high concentration of PM2.5-exposure group, indicating peak shift toward higher dose of PM2.5 group.

\section{Discussion}

Over the past decade, PM2.5 level in the atmosphere is increasing across China and nearby beyond, leading to the ensuing respiratory and cardiovascular disease at warp speed. More and more experimental studies have been conducted to investigate the toxic effects of PM2.5 on human health and their underlying mechanisms both in vivo and in vitro. To our knowledge, most in vitro studies employ one or several types of cells to be directly exposed to PM2.5 and explore possible toxic mechanisms. It is also 
noteworthy that macrophages may play an important role in the process of PM2.5 invading the human body. Pulmonary macrophages exposed to PM2.5 secrete a variety of cytokines that are released into the adjacent tissue and affect the proximity cells. However, among the toxic researches of PM2.5, there is a lack of evaluation of direct and indirect exposure models on bronchial epithelia cells. It has been worthwhile to figure out the different performance of epithelium interacted with PM2.5 directly or indirectly.

PM2.5 direct interaction on epithelium may strengthen the cytotoxic action. Our MTT results found that cell proliferation showed more significant inhibition with the direct exposure model compared with the indirect model. However, the viability of epithelia cells in co-cultured model didn't manifest a notable decrease in high concentration group. Exposure to environmental pollutants, allergens and pathogens can induce apoptosis of airway epithelial cells. Studies showed that PM exposure induced apoptosis by activating not only the tumor necrosis factor-alpha (TNF-alpha)-induced pathway, but also the mitochondrial pathway (Dagher et al. 2006). A recent research had demonstrated that a statistically significant association was demonstrated between the content of PAHs in PM2.5 and the rate of BEAS-2B apoptosis $(p<0.01)$ (Yang et al. 2016). Therefore, we speculated that the phagocytosis of macrophages may offset the cytotoxic effect of PM2.5, like PAHs in apoptosis,to maintain the epithelia function to some extent.

To further evaluate the effects of PM2.5 exposure on epithelial cells under different modes of interaction, we observed the oxidative stress transcription factor Nrf2 and the inflammatory response transcription factor NF-kB. In these two models, we found that different tendency occurred among NF-KB and downstream inflammatory cytokines, while there was no significant difference in the trend of Nrf2 in the two models. In the comparison of these two interaction models, the indirect stimulation model strengthen inflammation response of epithelial cells, especially under the effect of higher concentrations of PM2.5. According to the research, it has been demonstrated that PM2.5 exposure induce macrophage activation directly and initiate persistent inflammatory response via M1 polarization(Vogel et al. 2012). In general, macrophages can be polarized into M1/M2 distinct phenotypes: M1 macrophages which are mainly induced by lipopolysaccharide (LPS) considered to have higher antigen-presenting capacity and release a lot of pro-inflammatory cytokines. On the contrary, M2 macrophages mainly induced by interleukin-4 (IL4) act as anti-inflammatory ones (Zhu et al. 2015). The mounting evidence proved that PM2.5 drive macrophage into M1 phenotype (Zhao et al. 2016). According to a recent study, $25 \mu \mathrm{g} / \mathrm{ml}$ and higher concentration of PM2.5 derived from Beijing, which was identical with our sources induced the expression and secretion of pro-inflammatory cytokines by M1 macrophage. Whereas, different concentrations of PM2.5 didn't affect the expression of M2 macrophage markers (Zhong et al. 2019). Therefore, combining with our results, we proposed PM2.5-induced M1 macrophages release proinflammatory cytokines which could freely pass through the membrane hole in co-cultures cause a stronger inflammatory response on the epithelium than PM2.5 directly acting with epithelia.

It has been reported that the water-soluble fraction, which accounts for the major proportion of PM2.5 (Tan et al. 2017), induces more abnormal biological outcomes than water-insoluble PM2.5 components 
(Gutierrez-Castillo et al. 2006). Therefore, in addition to pro-inflammatory cytokines released from PM2.5induced M1 macrophages, the water-soluble fraction of PM2.5 such as metal(loid)s, ions and PAHs could freely pass through the membrane hole and trigger stronger inflammatory response on epithelia. Our studies indicated that the batch of PM2.5 we collected before contained high concentration of PAHs and metals such as $\mathrm{Zn}, \mathrm{Pb}, \mathrm{Mn}$ and $\mathrm{Cu}$. A former study has demonstrated a strong relationship between IL6/TNF-a secretion with the presence of $\mathrm{Cu}$ and $\mathrm{Zn}$ from anthropogenic sources PM2.5 in Mexicali (Osornio-Vargas et al. 2011). According to a recent investigation in which PM2.5 was sampled in 10 large Chinese cities over 1 year, IL-6 was significantly correlated with Pb, and endotoxin (Ma et al. 2019). Additional, it has reported that $\mathrm{Mn}$ and $\mathrm{Cr}$ were associated with IL-8 production in epithelial cells. Taken together, amid the process of PM2.5 stimulation, the presence of macrophage could strengthen the inflammation of epithelia.

\section{Conclusions}

Our data displayed as a comparative study on the mode of PM2.5 direct or indirect interaction with bronchial epithelial cells. We have found that macrophage involvement may protect epithelia from PM2.5 cytotoxic effect, while strengthen the inflammation response.

\section{Declarations}

\section{Declaration of Competing Interest}

The authors declare no potential conflicts of interest.

\section{Acknowledgment and funding sources}

This study was supported by the National Natural Science Foundation of China. (No. 81602832, 91643114 and 81973008$)$.

\section{Ethical Approval}

Not applicable.

\section{Consent to Participate}

The participants could withdraw from the study at any time without giving any reason.

\section{Consent to Publish}




\section{Authors Contributions}

Yan Wang: conceptualization, data analyses, original draft preparation; Xin Zuo: conceptualization, data analyses, material acquisition, original draft preparation; Fuyang Jiang: experimental operation, data analyses; Lin Hou: experimental verification, data analyses; Qiyue jiang: experimental verification, data analyses; Zhonghui Zhu: technical consulting, writing - review and editing, supervision; Lin Tian: conceptualization, funding acquisition, writing - review and editing, supervision.

\section{Availability of data and materials}

The datasets used and/or analyzed during the current study are available from the corresponding author on reasonable request.

\section{References}

1. Cho CC, Hsieh WY, Tsai CH, Chen CY, Chang HF, Lin CS (2018): In Vitro and In Vivo Experimental Studies of PM2.5 on Disease Progression. Int J Environ Res Public Health 15

2. Dagher Z, Garcon G, Billet S, Gosset P, Ledoux F, Courcot D, Aboukais A, Shirali P (2006): Activation of different pathways of apoptosis by air pollution particulate matter (PM2.5) in human epithelial lung cells (L132) in culture. Toxicology 225, 12-24

3. Falcon-Rodriguez Cl, Osornio-Vargas AR, Sada-Ovalle I, Segura-Medina P (2016): Aeroparticles, Composition, and Lung Diseases. Front Immunol 7, 3

4. Feng S, Gao D, Liao F, Zhou F, Wang X (2016): The health effects of ambient PM2.5 and potential mechanisms. Ecotoxicol Environ Saf 128, 67-74

5. Gutierrez-Castillo ME, Roubicek DA, Cebrian-Garcia ME, De Vizcaya-Ruiz A, Sordo-Cedeno M, Ostrosky-Wegman P (2006): Effect of chemical composition on the induction of DNA damage by urban airborne particulate matter. Environ Mol Mutagen 47, 199-211

6. Jeong S, Park SA, Park I, Kim P, Cho NH, Hyun JW, Hyun YM (2019): PM2.5 Exposure in the Respiratory System Induces Distinct Inflammatory Signaling in the Lung and the Liver of Mice. J Immunol Res 2019, 3486841

7. Kumar P, Kalaiarasan G, Porter AE, Pinna A, Klosowski MM, Demokritou P, Chung KF, Pain C, Arvind DK, Arcucci R, Adcock IM, Dilliway C (2021): An overview of methods of fine and ultrafine particle collection for physicochemical characterisation and toxicity assessments. Sci Total Environ 756, 143553

8. Li Q, Sun J, Chen X, Li S, Wang Y, Xu C, Zhao J, Zhu Z, Tian L (2020): Integrative characterization of fine particulate matter-induced chronic obstructive pulmonary disease in mice. Sci Total Environ 706, 
9. Ma H, Li J, Wan C, Liang Y, Zhang X, Dong G, Hu L, Yang B, Zeng X, Su T, Lu S, Chen S, Khorram MS, Sheng G, Wang X, Mai B, Yu Z, Zhang G (2019): Inflammation Response of Water-Soluble Fractions in Atmospheric Fine Particulates: A Seasonal Observation in 10 Large Chinese Cities. Environ Sci Technol 53, 3782-3790

10. Osornio-Vargas AR, Serrano J, Rojas-Bracho L, Miranda J, Garcia-Cuellar C, Reyna MA, Flores G, Zuk M, Quintero M, Vazquez I, Sanchez-Perez Y, Lopez T, Rosas I (2011): In vitro biological effects of airborne PM(2).(5) and PM(1)(0) from a semi-desert city on the Mexico-US border. Chemosphere 83, 618-26

11. Tan J, Zhang L, Zhou X, Duan J, Li Y, Hu J, He K (2017): Chemical characteristics and source apportionment of PM2.5 in Lanzhou, China. Sci Total Environ 601-602, 1743-1752

12. Tonelli C, Chio IIC, Tuveson DA (2018): Transcriptional Regulation by Nrf2. Antioxid Redox Signal 29, 1727-1745

13. Vogel CF, Garcia J, Wu D, Mitchell DC, Zhang Y, Kado NY, Wong P, Trujillo DA, Lollies A, Bennet D, Schenker MB, Mitloehner FM (2012): Activation of inflammatory responses in human U937 macrophages by particulate matter collected from dairy farms: an in vitro expression analysis of proinflammatory markers. Environ Health 11, 17

14. Yang L, Liu G, Lin Z, Wang Y, He H, Liu T, Kamp DW (2016): Pro-inflammatory response and oxidative stress induced by specific components in ambient particulate matter in human bronchial epithelial cells. Environ Toxicol 31, 923-36

15. Zhao Q, Chen H, Yang T, Rui W, Liu F, Zhang F, Zhao Y, Ding W (2016): Direct effects of airborne PM2.5 exposure on macrophage polarizations. Biochim Biophys Acta 1860, 2835-43

16. Zhong Y, Liao J, Hu Y, Wang Y, Sun C, Zhang C, Wang G (2019): PM2.5 Upregulates MicroRNA-146a3p and Induces M1 Polarization in RAW264.7 Cells by Targeting Sirtuin1. Int J Med Sci 16, 384-393

17. Zhu L, Zhao Q, Yang T, Ding W, Zhao Y (2015): Cellular metabolism and macrophage functional polarization. Int Rev Immunol 34, 82-100

18. Zhu Z, Chen X, Sun J, Li Q, Lian X, Li S, Wang Y, Tian L (2019): Inhibition of nuclear thioredoxin aggregation attenuates PM2.5-induced NF-kappaB activation and pro-inflammatory responses. Free Radic Biol Med 130, 206-214

\section{Figures}




\section{Composition analysis of $\mathbf{P M}_{2.5}$}

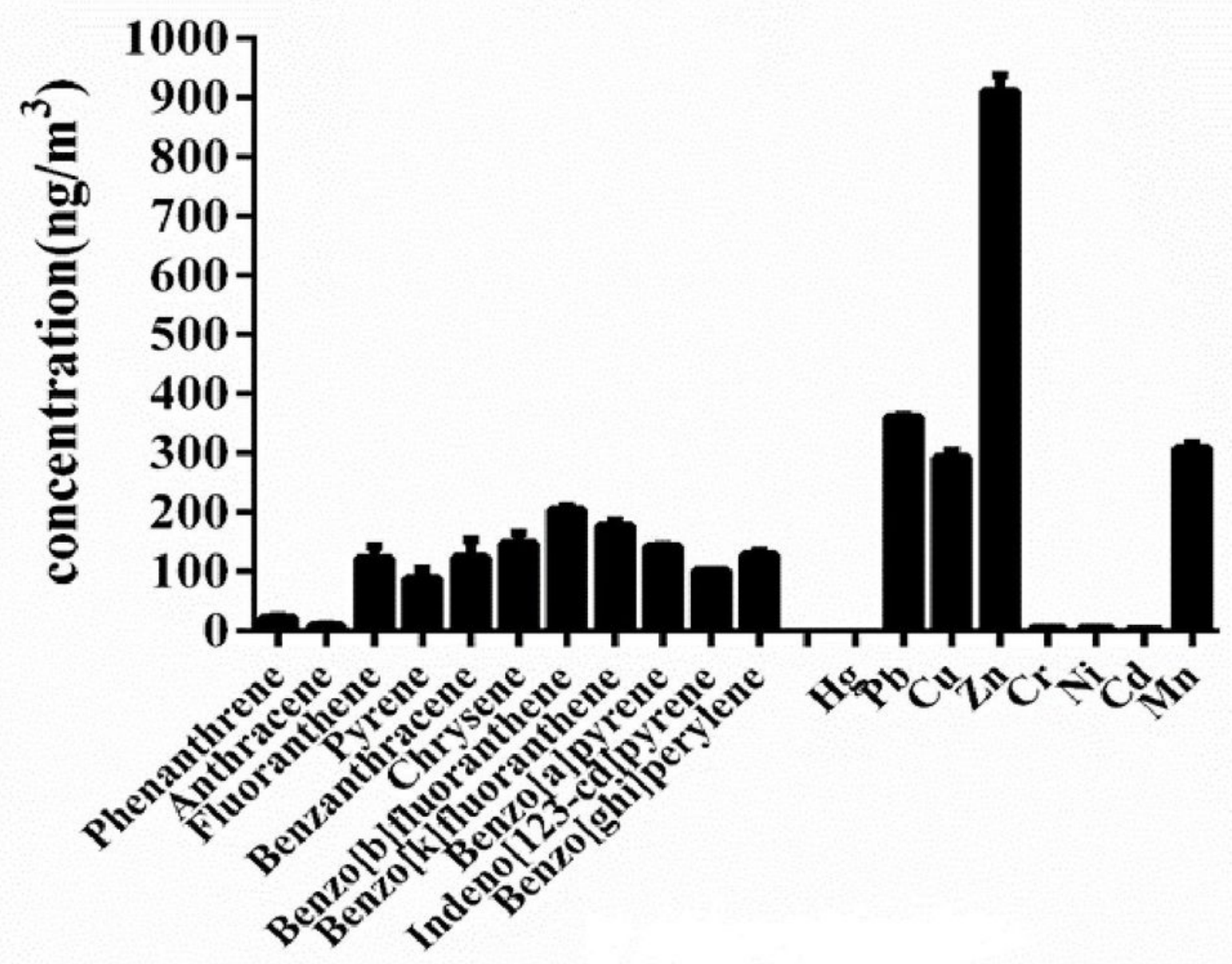

Figure 1

Gas chromatography/mass spectrometry analysis of the aromatic compounds and inorganic elements in collecting PM2.5 particles. 


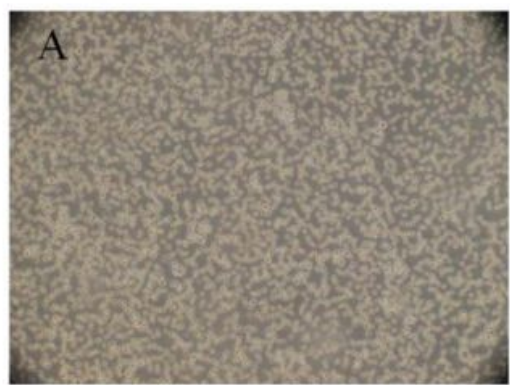

C

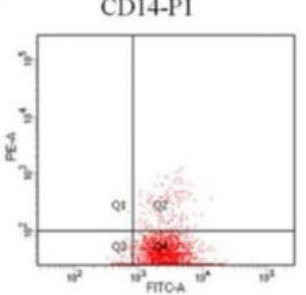

CD68-PI

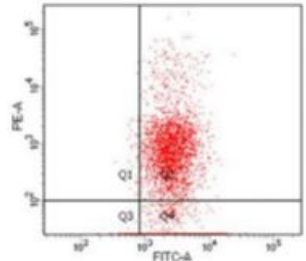

THP-1
CD14-P2

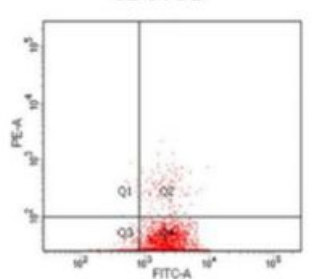

CD68-P2

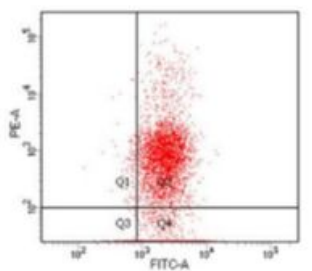

THP-1- M $\varphi$
D

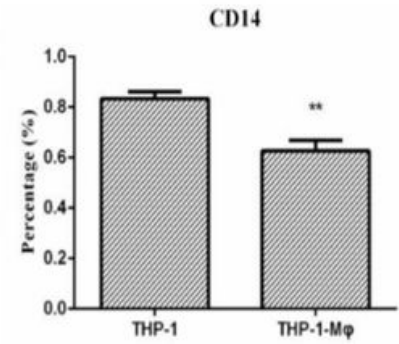

$\mathbf{E}$

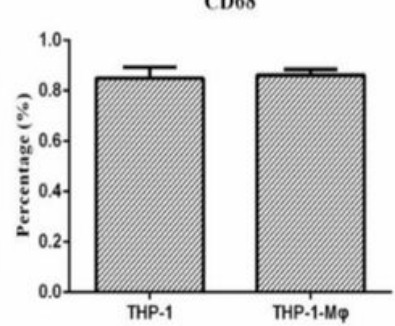

Figure 2

Differentiation of THP-1 cells to turn into macrophages. (A) The cells were treated with RPMI1640 complete medium (THP-1 group), $50 \mathrm{ng} / \mathrm{mL}$ PMA for $48 \mathrm{~h}$. Morphological changes were visualized by a phase contrast inverted microscope under $10 \mathrm{x}$ magnification. (B) Flow cytometry analyzed the expression of CD14 and CD68 by THP-1 cells and THP-1 macrophages.

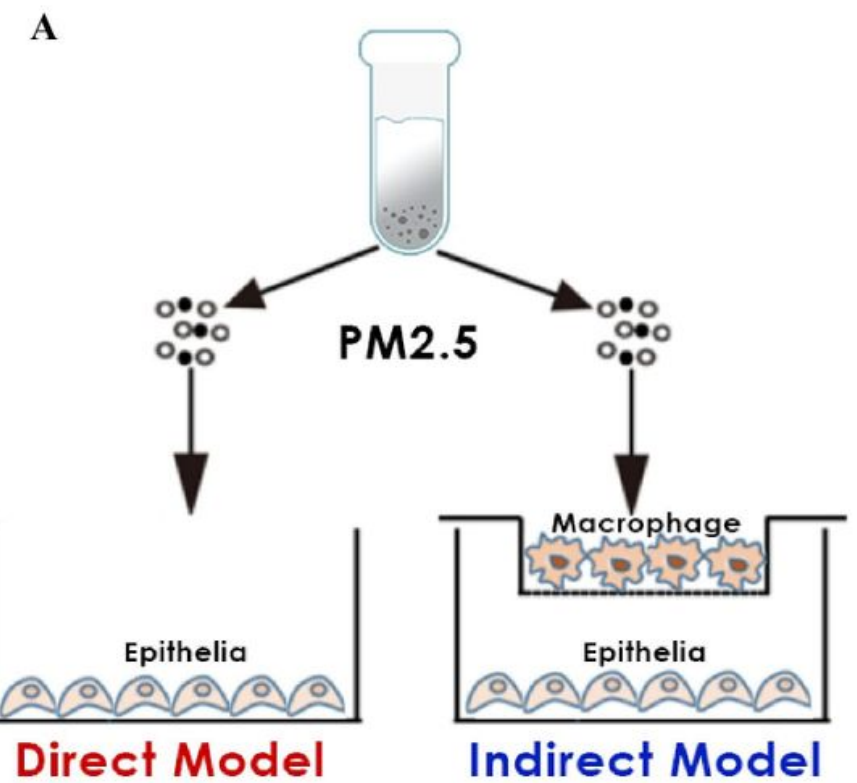

B

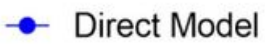

- Indirect Model

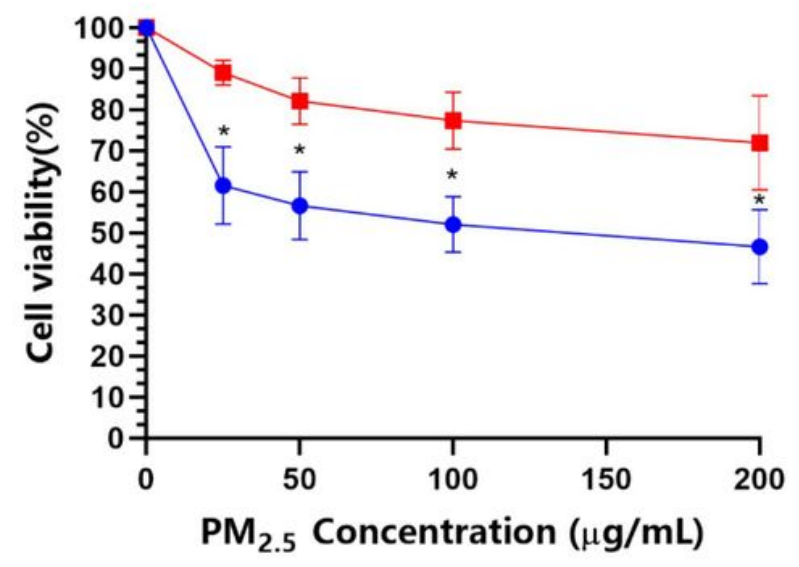

Figure 3 
(A) Schematic illustration of the experimental design. (B) MTT analysis of cell viability. Each concentration was tested in three wells and the experiments were repeated three times. ${ }^{*} P<0.05$ vs. indirect model.

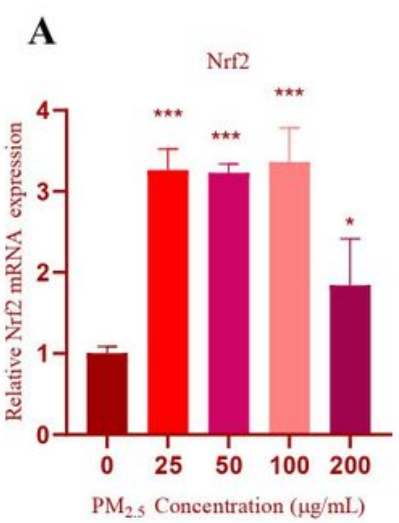

$\mathbf{F}$

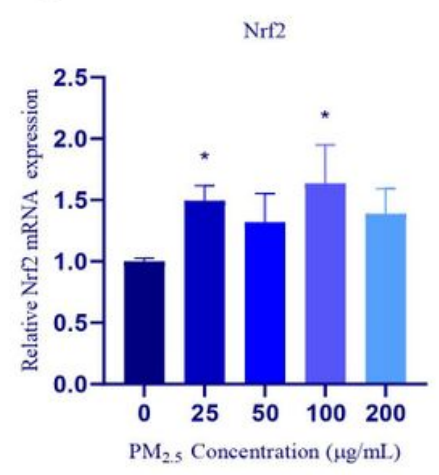

B

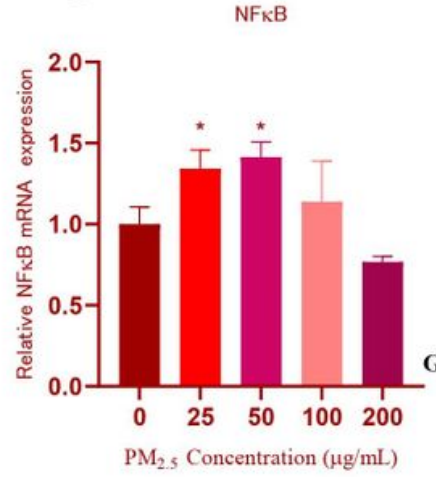

G

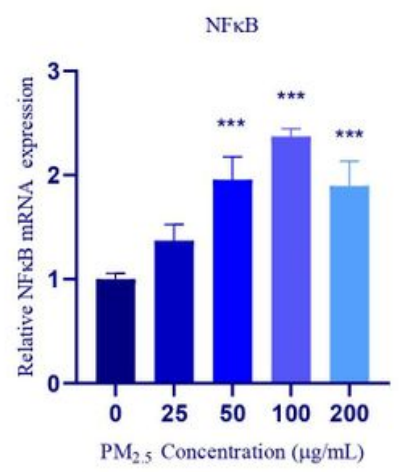

C

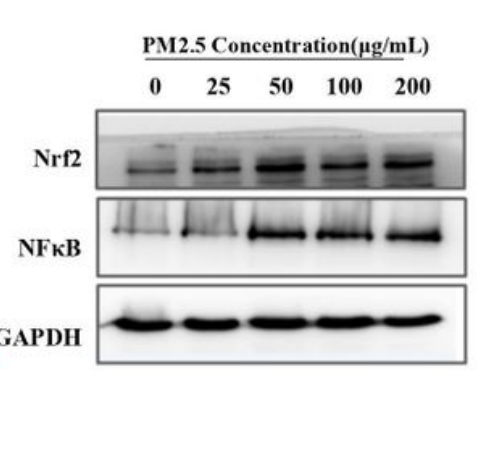

$\mathbf{H}$

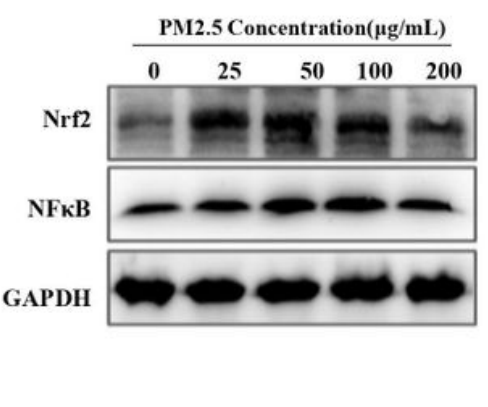

D
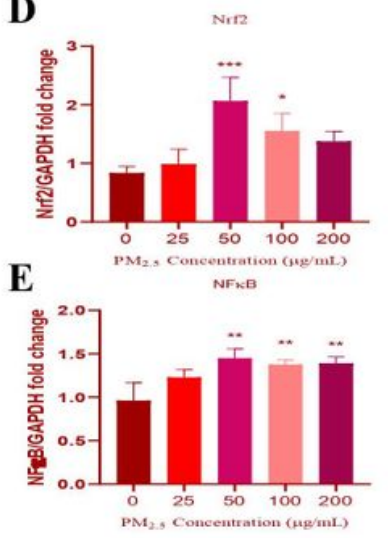

I
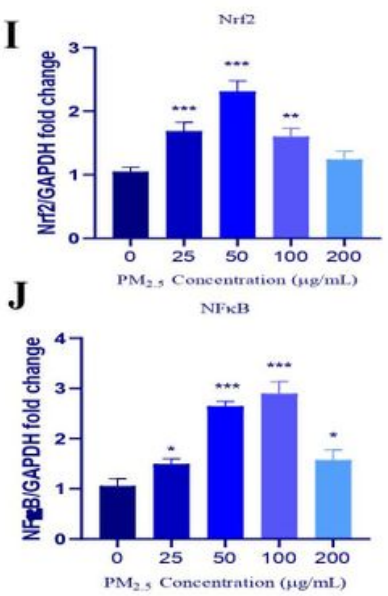

Figure 4

Changes in gene and protein expression of transcription factors Nrf2 and NF-kB in both models with the red of direct model and the blue of indirect model. ${ }^{\star} P<0.05$ vs. no $P M 2.5(0 \mu \mathrm{g} / \mathrm{mL}),{ }^{\star \star} P<0.01 \mathrm{vs}$. no PM2.5 $(0 \mu \mathrm{g} / \mathrm{mL}),{ }^{\star \star *} \mathrm{P}<0.001$ vs. no PM2.5 $(0 \mu \mathrm{g} / \mathrm{mL})$. 
A

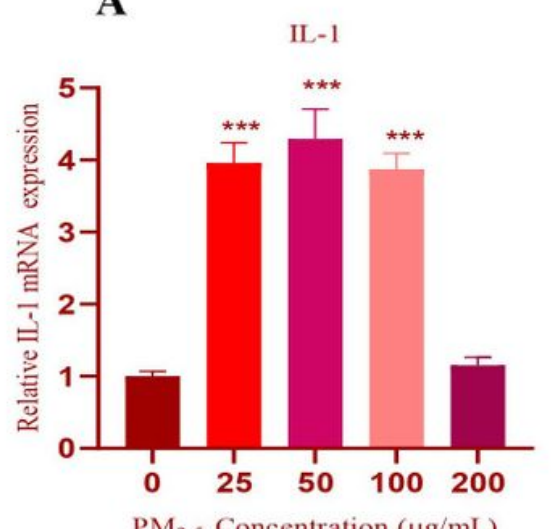

D

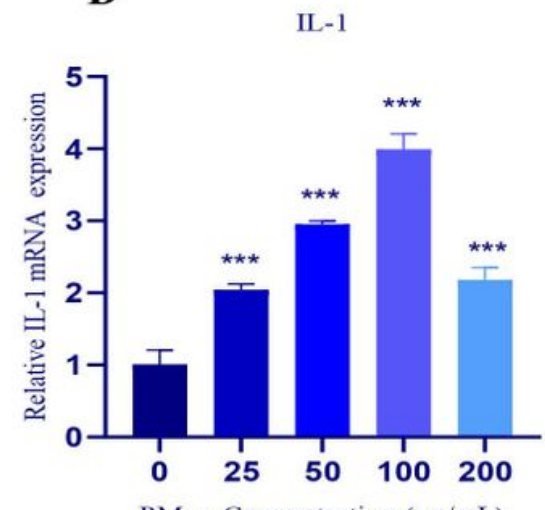

$\mathrm{PM}_{2.5}$ Concentration $(\mu \mathrm{g} / \mathrm{mL})$
B

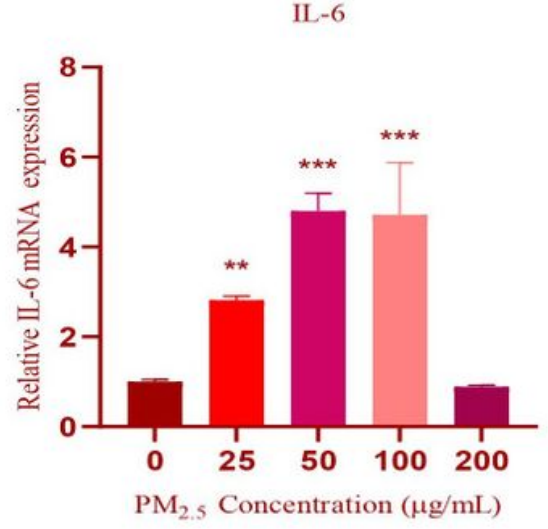

E

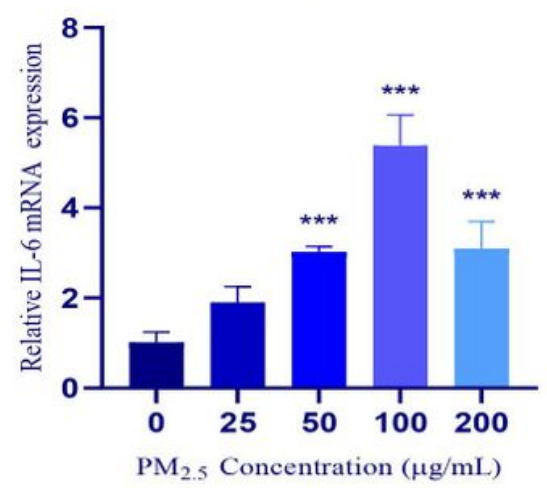

C

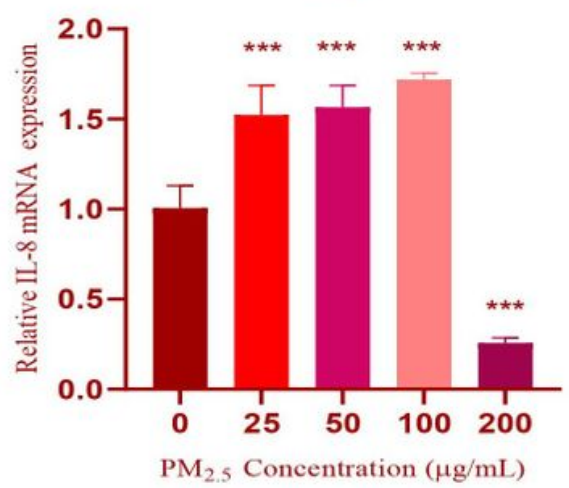

F

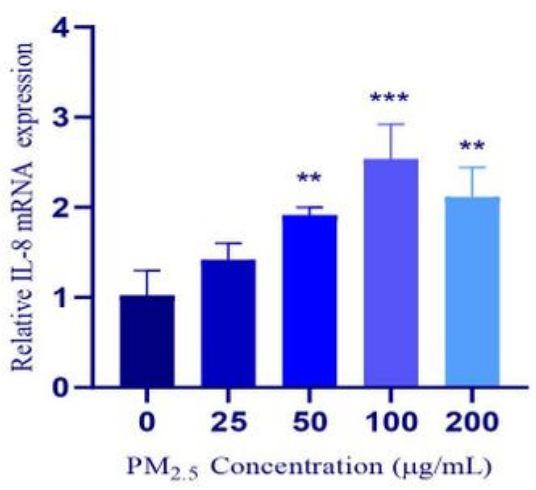

Figure 5

Changes in gene expression of inflammation factors IL-1, IL-6, IL-8 in both models with the red of direct model and the blue of indirect model. ${ }^{\star} * \mathrm{P}<0.01$ vs. no $\mathrm{PM} 2.5(0 \mu \mathrm{g} / \mathrm{mL})$, ${ }^{\star \star *} \mathrm{P}<0.001$ vs. no $\mathrm{PM} 2.5(0$ $\mu \mathrm{g} / \mathrm{mL})$. 